\title{
Bayesian Spatiotemporal Gaussian Process for Short-term Load Forecasting Using Combined Transportation and Electricity Data
}

\author{
MOSTAFA GILANIFAR, HUI WANG, and EREN ERMAN OZGUVEN, Florida State University \\ YUXUN ZHOU, University of California, Berkeley \\ REZA ARGHANDEH, Western Norway University of Applied Sciences
}

\begin{abstract}
Smart cities can be viewed as large-scale Cyber-Physical Systems (CPS) where different sensors and devices record the cyber and physical indicators of the city systems. The collected data are used for improving urban life by offering services such as accurate electric load forecasting, and more efficient traffic management. Traditional monitoring for electricity and transportation networks generally do not provide full observability due to their limited coverage as well as high implementation and maintenance costs. For example, continuous traffic data collection is mostly limited to major highways only in big cities, whereas local roadways are usually covered once or twice a year. Also, there are no high-fidelity and real-time electric monitoring systems in all parts of power distribution networks. Combining the limited data from each of the urban systems together (e.g., electricity, transportation, environment, etc.) provides a better picture of the energy flow in a city. Furthermore, a city should be considered as a collection of the layers of tangled infrastructure networks, which connects people, places, and resources. Therefore, the study of traffic or electricity consumption forecasting should go beyond the transportation and electricity networks and merge with each other and even with other city networks such as environmental networks. As such, this article proposes a Bayesian spatiotemporal Gaussian Process model that employs the most informative spatiotemporal interdependency among different interconnected networks (in this case, electricity, transportation, and weather). The proposed load forecasting method is compared with other state-of-the-art methods using real-life data obtained from the City of Tallahassee in Florida. Results show that the proposed Bayesian spatiotemporal Gaussian Process model outperforms state-of-the-art methods.
\end{abstract}

CCS Concepts: • Information systems $\rightarrow$ Sensor networks;

Additional Key Words and Phrases: Bayesian spatiotemporal gaussian process, spatiotemporal dependencies, traffic load forecasting, electric load forecasting

This research is partly supported by U.S. NSF award 1640587 and NSF grant CMMI-1434411.

Authors' addresses: M. Gilanifar and H. Wang, Industrial and Manufacturing Eng. Dept., Florida State University (FSU) (2525 Pottsdamer St., Tallahassee, FL 32310-6046); emails: mostafa.gilanifar@gmail.com, hwang10@eng.famu.fsu.edu; E. E. Ozguven, Civil and Environmental Eng. Dept., FSU, (2525 Pottsdamer St., Tallahassee, FL 32310-6046); email: eozguven@fsu.edu; Y. Zhou, Electrical Eng. and Computer Science Dept., University of California Berkeley, (2626 Hearst Ave, Berkeley, CA); email: yxzhou@berkeley.edu; R. Arghandeh, Department of Computing, Mathematics, and Physics, Western Norway University of Applied Sciences (Høgskulen på Vestlandet, Postbox 7030, 5020 Bergen, Norway); email: arghandehr@gmail.com.

Permission to make digital or hard copies of all or part of this work for personal or classroom use is granted without fee provided that copies are not made or distributed for profit or commercial advantage and that copies bear this notice and the full citation on the first page. Copyrights for components of this work owned by others than ACM must be honored. Abstracting with credit is permitted. To copy otherwise, or republish, to post on servers or to redistribute to lists, requires prior specific permission and/or a fee. Request permissions from permissions@acm.org.

(C) 2019 Association for Computing Machinery.

2378-962X/2019/10-ART2 \$15.00

https://doi.org/10.1145/3300185

ACM Transactions on Cyber-Physical Systems, Vol. 4, No. 1, Article 2. Publication date: October 2019. 


\section{ACM Reference format:}

Mostafa Gilanifar, Hui Wang, Eren Erman Ozguven, Yuxun Zhou, and Reza Arghandeh. 2019. Bayesian Spatiotemporal Gaussian Process for Short-term Load Forecasting Using Combined Transportation and Electricity Data. ACM Trans. Cyber-Phys. Syst. 4, 1, Article 2 (October 2019), 25 pages.

https://doi.org/10.1145/3300185

\section{INTRODUCTION}

The recent advances related to the monitoring of cyber-physical systems has increased the ability to collect, analyze, and utilize massive amounts of data for the infrastructure networks operation of cities [Zheng et al. 2014]. Numerous data-driven decision support methods have been proposed to capture the flow of people, energy, and commodities in cities [Zhang et al. 2017]. However, most of the available methods are ad hoc and dedicated to an individual urban network and suffer from data sparsity [Zheng et al. 2014]. This is due to the complexity and the high cost of data mining from large-scale spatial and temporal networks. For example, continuous traffic data collection is mostly limited to major highways only in many cities, whereas secondary and local roadways are usually covered once or twice a year and there are no high-fidelity and real-time monitoring systems in all parts of power distribution networks.

To avoid data sparsity and scarcity issues in any urban network (e.g., electricity network), multinetwork spatiotemporal data fusion can be helpful. The organic interdependency among different urban networks shades more light to the lack of data in each one of those networks. The multinetwork data fusion also reduces the inference uncertainty due to the lack of information. In the case of the traffic load forecasting, most of the state-of-the-art forecasting methods have solely relied on historical traffic data. For example, Work et al. [2010] used the first-order LighthillWhiteham-Richards (LWR) model for macroscopic modeling purposes for freeways. They used GPS data and a Monte Carlo-based ensemble Kalman Filters (KFs) for estimating the traffic states. Similarly, Herrera et al. [2010] used GPS data to provide accurate measurement of the traffic speed. Hofleitner et al. [2012], on the other hand, constructed a Dynamic Bayesian Network (DBN) to perform a real-time estimation of traffic dynamics with the help of the Expected Maximization (EM) algorithm, whereas Sen et al. [2013] tried to estimate traffic density and speed in bi-directional traffic using image processing tools. Although some researchers have tried to use different datasets, their data always belonged to single-type data. For instance, Deng et al. [2013] and Xing et al. [2013] used loop detector counts, AVI Bluetooth travel time readings, and GPS samples, which are used to predict traffic states for freeways and develop traffic sensor network design, respectively. Recently, Nantes et al. [2016] implemented an LWR model focusing on macroscopic traffic states for urban corridors with an extended KFs approach. They also used loop detector counts, AVI Bluetooth, and GPS data for a real-time traffic prediction model. However, all these studies have only used transportation data to estimate traffic states or counts.

In an electricity load forecasting area, most of the available methods also performed the load forecasting by only using the historical electricity network data. Ghofrani et al. [2011] studied the short-term load forecasting in an individual household level using a Kalman filter estimator. Humeau et al. [2013] improved the electricity load forecasting by using the statistical relations between time series from different smart meters. Chaouch [2014] performed the short-term forecasting for residential customers with a functional time-series method. More recently, Hsiao [2015] proposed a model for the very short-term residential loads based on the context information from smart meter data. In addition, Kong et al. [2017] showed that forecasting error could be decreased by using appliance measurements in their data.

The traffic and electricity consumption are highly dependent on the weather. This was the source of inspiration for some researchers to characterize the dependency of the weather forecast and 
electric load or traffic load forecast. For example, the impact of weather, such as the temperature, on the road safety has been shown in El-Faouzi [2010]. Cools et al. [2010] presented the effects of weather on the frequency of trips and travel behavior. More recently, Rudloff et al. [2015] quantified the weather-related effects on transportation mode and purpose of transportation demand. All of these researchers tried to prove the effects of weather information on traffic and mobility. However, some previous studies have shown the impact of weather on the electricity network. For example, Wi et al. [2012] proposed a feature selection process implementing weather data for improving the efficiency of the fuzzy polynomial regression method. Ruzic et al. [2003] developed a forecasting algorithm based on a regression model that suitable for weather data in short-term load forecasting. Chow et al. [1996] presented a nonlinear Box and Jenkins approach for load forecasting based on neural weather compensation. Taylor and Buizza [2002] used the weather ensemble predictions for load forecasting for 10 days ahead, and Chen et al. [1992] proposed an artificial neural network (ANN) model for forecasting the weather-sensitive loads for an entire week. In addition, Davis [1959] described a method to statistically analyze the relationship between weather and electricity demand, whereas Fay et al. [2010] considered the effect of errors available in weather forecasting into the short-term load forecasting models.

As such, the previous studies tried either to make predictions by using data from a single network or by only fusing the weather data into that network's historical data for forecasting purposes. However, cities are tangled multilayer systems that connect people, places, and resources. These tangled systems include electricity, transportation, water, gas, social networks, and so on, shaping the urban life fabric. For instance, there is a logical relationship between traffic and electricity usage in the morning and evening rush hours, where people are leaving their houses and going to work and vice versa around a similar time. In other words, when people are on the roadways, there is more traffic and less electricity consumption in the households. Therefore, the spatiotemporal patterns of mobility that reflect the presence of people in different locations can shed more light on the electricity consumption by providing prior information.

A few studies have been conducted on the interdependencies between transportation and electricity networks. However, the majority of them are focused on the electric vehicles charging schemes and transportation electrification, see Marti [2006] and Kelly et al. [2015]. Also, Ostermann et al. [2016] presented an energy management system that provides energy-managementas-a-service for electric vehicle fleet operators. More recently, Aparicio et al. [2014] tried to express the correlation between the road traffic data and electric load forecasting. They used the Pearson's correlation coefficient for finding the correlation between these two networks. Although they considered the aggregated traffic jam length for the main roads in a major city in Belgium, this cannot wholly represent the mobility and its impact on the electric network. That is, in many cities, the traffic jams are due to people going from their origins to destinations, which may not be related at all with the power consumption on that specific roadway section. They neither considered the vehicles in uncongested streets nor the spatial dependencies between the data. To the best of authors' knowledge, the interdependencies and interactions between electricity and transportation networks in the context of urban mobility have not been appropriately addressed in the literature.

This study is using the Bayesian Spatiotemporal Gaussian Process to show that the mobility should go beyond transportation and power systems and merge with other infrastructure and information networks. This article is the continuation of authors ongoing study on urban mobility in multi-networked environments [Madhavi et al. 2017, 2019; Cordova et al. 2018; Pinzan et al. 2018; K. S. et al. 2018]. This article will address the following research gaps:

- There is a need to study the interdependency of household electric consumption and urban mobility that is a reflection of the city inhabitant's real-life movement patterns along 
with meteorological information to improve the traffic load forecasting, and electricity load forecasting.

- There is also a lack of systematic studies on the method selection and comparison for fusing urban mobility data along with meteorological data to improve the electric load forecasting. This article proposes a Bayesian Spatiotemporal Gaussian Process model to solve the data fusion problem for improving load forecasting and demonstrates its effectiveness by comparing it with other state-of-the-art data fusion methods.

The rest of this article is organized as follows: Section 2 explains the multi-source spatiotemporal Gaussian Process model for traffic/electric load forecasting followed by presenting several forecasting performance indices and some state-of-the-art forecasting methods. Section 3 describes the real-world dataset, and Section 4 provides the results and discussions of the proposed framework. Finally, Section 5 finalizes the article with a concise conclusion.

\section{METHODOLOGY}

Suppose that we have observed response variable $R(s, t)$ with the indexes of $s$ for location and $t$ for time. If we have $n$ spatial locations $s_{1}, \ldots, s_{n}$ and $\tau$ different time points, then our observed response variable $R(s, t)$ can be formulated as

$$
R(s, t)=\left(R\left(s_{1}, t\right), \ldots, R\left(s_{n}, t\right)\right)^{\prime}, t=1, \ldots, \tau .
$$

Like any model fitting, observed response variable $(R(s, t))$ can be shown as predicted term $(\hat{R}(s, t))$ with some errors. So we can write

$$
R(s, t)=\hat{R}(s, t)+\epsilon(s, t),
$$

where $\hat{R}(s, t)$ is called mean process of the model corresponding to $R(s, t)$. All the $R(s, t), \hat{R}(s, t)$, and $\epsilon(s, t)$ are column vectors with $l \times 1$ dimension where $l=\tau \times n ; \tau$ is the time points that we have and $n$ is the amount of spatial locations. The term $\epsilon(s, t) \sim N\left(0, \sigma_{\epsilon}^{2} I_{l}\right)$ is independent and identically distributed (IID) normal, which is called noise error. $\sigma_{\epsilon}^{2}$ works as our variance, and $I_{l}$ is the $l \times l$ identity matrix.

\subsection{Bayesian Spatio-temporal Gaussian Process Model}

The mean process of the model $\hat{R}(s, t)$ can include a Trend term with some correlation errors $(\eta)$ that characterizes spatiotemporal dependencies among data. The mean process of the model $\hat{R}(s, t)$ can be postulated as follows:

$$
\hat{R}(s, t)=\operatorname{Trend}(s, t)+\eta(s, t) .
$$

The term $\eta(s, t)$ is normally distributed with mean 0 and variance-covariance matrix of $\Sigma_{n}$ $\left(\eta(s, t) \sim N\left(0, \Sigma_{\eta}\right)\right)$ for any $t=1, \ldots, \tau$ and $s=s_{1}, \ldots, s_{n}$. Moreover, $\eta(s, t)$ is a Gaussian Process that is called spatially correlated error that is independent in time. The variance-covariance matrix is $\Sigma_{\eta}=\sigma_{\eta}^{2} S_{\eta}=\sigma_{\eta}^{2} \kappa\left(s_{i}, s_{j} ; \phi\right)$ for any $i=1, \ldots, n$ and $j=1, \ldots, n$. For spatial correlation function $\left(\kappa\left(s_{i}, s_{j} ; \phi\right)\right)$, we use the exponential correlation function [Matern 1986] in this article. The exponential correlation function is defined as

$$
\kappa\left(s_{i}, s_{j} ; \phi\right)=\exp \left(-\phi\left\|s_{i}-s_{j}\right\|\right), \phi>0,
$$

where $\left\|s_{i}-s_{j}\right\|$ is the distance between site $s_{i}$ and $s_{j}$ and $\phi$ is the spatial decay parameter, which is specified by a Gamma distribution as a prior distribution [Bakar 2012].

A simple explanation of the Gaussian Process $\eta$ in Equation (3) is when we want to forecast in one location $\left(s^{\prime}\right)$ at one-step ahead $(t+1)$. First, our Gaussian Process $(\eta)$ predicts for one-step ahead in those locations that already have the data, and once it predicted at one-step ahead $(t+1)$, it tries to predict for the new location $\left(s^{\prime}\right)$ at the same time. For better clarification, Figure 1 shows 


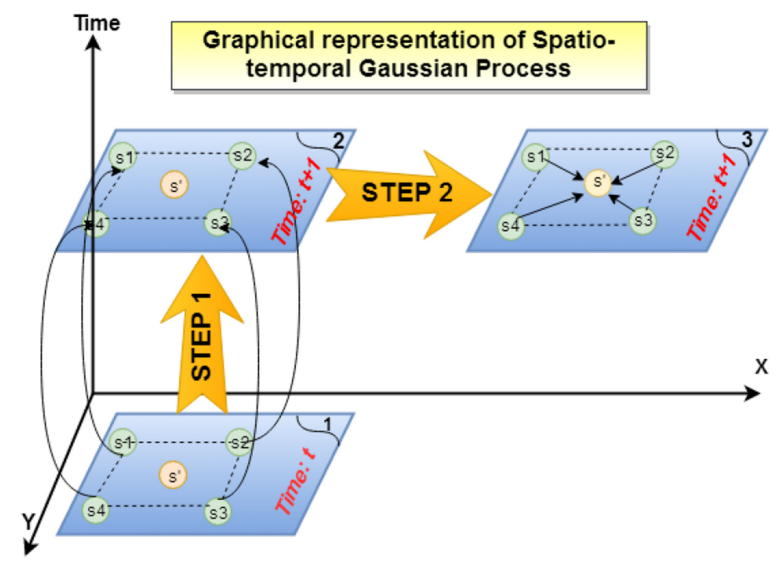

Fig. 1. Graphical representation of spatiotemporal Gaussian Process $(\eta(s, t))$.

the graphical representation of the spatiotemporal Gaussian Process $\eta$ when, for instance, we want to forecast the load for a specific location $\left(s^{\prime}\right)$. So, for the specific location, the spatial-temporal Gaussian Process considers temporal dependencies to obtain the load in one-step ahead $(t+1)$ and also, for one specific time, it considers spatial variations to obtain the load in the new location. Also, this Gaussian Process cannot consider the variations from different locations in different time steps directly. As we stated before, $\eta$ is independent in time, but, indirectly in two different steps, it considers the dependencies of different locations in different time steps (Figure 1).

The "Trend" in Equation (3) captures the mean shift or trending patterns in the data. The Trend is commonly assumed as a linear model of variables that are correlated to the response variable. By assuming a linear regression function, the "Trend" can be expanded by

$$
\operatorname{Trend}(s, t)=X(s, t) \vec{\beta},
$$

where $\vec{\beta}$ is coefficients of input variables and $X$ is the input variables that can include electric, environmental, and traffic variables. In this article, there are two different goals including traffic load forecasting and electric load forecasting. According to these goals, the Trend is formulated as follows:

2.1.1 Traffic Load Forecasting. According to the input variables, traffic load forecasting is categorized in four different scenarios as below

1. Only Previous Traffic Load:

$$
\operatorname{Trend}(s, t)=\operatorname{Tr}(s, t)=\left(\sum_{i=1}^{p} \beta_{i} \operatorname{Tr}(s, t-p)\right),
$$

where $T r$ is the traffic load and $p$ represents the number of time lags in traffic load time series.

2. Previous Traffic Load + Environmental Variables:

$$
\operatorname{Trend}(s, t)=\operatorname{Tr}(s, t)=\left(\sum_{i=1}^{p} \beta_{i} \operatorname{Tr}(s, t-p)\right)+\beta_{p+1} T(s, t)+\beta_{p+2} H(s, t)+\beta_{p+3} S(s, t),
$$

where $T, H$, and $S$ are temperature, humidity, and solar radiation, respectively. 
3. Previous Traffic Load + Electric Variable:

$$
\operatorname{Trend}(s, t)=\operatorname{Tr}(s, t)=\left(\sum_{i=1}^{p} \beta_{i} \operatorname{Tr}(s, t-p)\right)+\beta_{p+1} E(s, t),
$$

where $E$ is the electric consumption of all households in a residential community near the traffic load measurement.

4. Previous Traffic Load + Environmental Variables + Electric Variable:

$$
\begin{aligned}
\operatorname{Trend}(s, t)=\operatorname{Tr}(s, t)= & \left(\sum_{i=1}^{p} \beta_{i} \operatorname{Tr}(s, t-p)\right)+\beta_{p+1} T(s, t) \\
& +\beta_{p+2} H(s, t)+\beta_{p+3} S(s, t)+\beta_{p+4} E(s, t) .
\end{aligned}
$$

In this scenario, all the previous traffic load, environmental variables and electric consumption data are used in the Trend for traffic load forecasting.

2.1.2 Electric Load Forecasting. Electric load forecasting similar to the traffic load forecasting is categorized into four scenarios based on the input variables as below

1. Only Previous Electric Load:

$$
\operatorname{Trend}(s, t)=E(s, t)=\left(\sum_{i=1}^{p} \beta_{i} E(s, t-p)\right),
$$

where $E(s, t)$ is the electric consumption for location (house) $s$ and time $t$. As it is shown, in this scenario, the electric consumption is forecasted by ony electric consumption in the previous times.

2. Previous Electric Load + Environmental Variables:

$$
\operatorname{Trend}(s, t)=E(s, t)=\left(\sum_{i=1}^{p} \beta_{i} E(s, t-p)\right)+\beta_{p+1} T(s, t)+\beta_{p+2} H(s, t)+\beta_{p+3} S(s, t),
$$

where the same as before, $T, H$, and $S$ represent the temperature, humidity, and solar radiation.

3. Previous Electric Load + Traffic Counts:

$$
\operatorname{Trend}(s, t)=E(s, t)=\left(\sum_{i=1}^{p} \beta_{i} E(s, t-p)\right)+\beta_{p+1} \operatorname{Tr}(s, t),
$$

where $\operatorname{Tr}$ is the traffic load near the residential community.

4. Previous Electric Load + Environmental Variables + Traffic Counts:

$$
\begin{aligned}
\operatorname{Trend}(s, t)=E(s, t)= & \left(\sum_{i=1}^{p} \beta_{i} E(s, t-p)\right)+\beta_{p+1} T(s, t) \\
& +\beta_{p+2} H(s, t)+\beta_{p+3} S(s, t)+\beta_{p+4} \operatorname{Tr}(s, t) .
\end{aligned}
$$

In this scenario, all the previous electric load observations along with the environmental variables and traffic load near the residential community are used for the electric load forecasting.

The time lag $p$ in the time-series model in all above equations is determined by the Autocorrelation Function (ACF), and Partial Autocorrelation Function (PACF) figures to find how many previous observations are needed in the model. Also, we need to confirm that the time series is stationary. Autocorrelation reflects the linear dependence of a variable with itself at two points in time. For stationary processes, autocorrelation between any two observations only depends on the 
time lag "k" between them. Usually, when the ACF declines rapidly, it shows that the time series is stationary. On the other hand, a partial autocorrelation is the amount of correlation between a variable and a lag of itself that is not explained by correlations at all lower-order-lags. The autocorrelation of a time series $\mathrm{Y}$ at lag 1 is the coefficient of correlation between Yt and Yt-1. The partial autocorrelations at all lags can be computed by fitting a succession of autoregressive models with increasing numbers of lags. In particular, the partial autocorrelation at a lag " $\mathrm{k}$ " is equal to the estimated $\mathrm{AR}(\mathrm{k})$ coefficient in an autoregressive model with " $\mathrm{k}$ " terms, i.e., a multiple regression model, in which Y is regressed on LAG(Y,1), LAG(Y,2), and so on, up to LAG(Y,k). Thus, the inspection of the PACF can determine how many AR terms are necessary to explain the autocorrelation pattern in a time series. If the partial autocorrelation is significant at the lag " $k$ " but insignificant at any higher order lags, i.e., if the PACF "cuts off" at the lag "k," then the autoregressive model can be fitted for an of order "k."

$R(s, t)$ can be parametrized by the four parameters $\theta=\left(\beta, \sigma_{\eta}^{2}, \phi, \sigma_{\epsilon}^{2}\right)^{\prime}$, which can be estimated by using a Bayesian hierarchical structure. The goal is to obtain the spatial and temporal dependencies available in the dataset and to be able to make a forecast $R(s, t+1)$ at desired spatial location $s$ and one-step time ahead $t+1$.

According to Gelfand [2012], any stochastic model is well suited for incorporating the prior knowledge, allowing it to be inserted at various levels of the modeling. Thus, a Bayesian spatiotemporal model can be formulated under a hierarchical framework, which can be widened by suitable marginalization/integration. The advantage of the hierarchical form lies in the convenience of the specification, ease of interpretation, and, often, in the facilitation of model fitting. Furthermore, by recognizing the uncertainty in the model parameters, uncertainty is properly propagated to inference arising from the model [Gelfand 2012].

Specifically, $R(s, t)$ in Equation (2) yields a marginal covariance matrix of the form $\operatorname{Cov}=\Sigma_{\eta}+$ $\sigma_{\epsilon}^{2} I_{l}$, which is a summation of covariance of spatially correlated error $(\eta)$ and noise error $(\epsilon)$. The likelihood of $R(s, t)$ is given by $R(s, t) \mid \eta, \theta N\left(\operatorname{Trend}(s, t), \Sigma_{\eta}+\sigma_{\epsilon}^{2} I_{l}\right)$. The $R(s, t)$ can be given in a hierarchical setup by considering $R(s, t)$ conditioned on the spatially correlated error $(\eta)$ and $\theta$, which $(\eta)$ further relies on the parameter $\theta$ with a prior, i.e.,

First Hierarchy: $\quad[R(s, t) \mid \eta(s, t), \theta]$

Second Hierarchy: $\quad[\eta(s, t) \mid \theta]$

Third Hierarchy: $[\theta]$

where $(\eta)$ and $\theta$ follow distributions as discussed before. According to the above hierarchical presentation, we can fit the model as $p(R \mid \theta) p(\theta)$ or as marginal model such as $p(R \mid \eta, \theta) p(\eta \mid \theta) p(\theta)$. Fitting the hierarchical model has better computation performance due to a lower dimension and more stable in terms of matrix inversion needed for sampling and likelihood evaluation [Gelfand 2012]. The hierarchical model can greatly facilitate derivation of the marginal distribution of the response (electricity load or traffic), which estimates the probabilistic performance of the model.

If we want to forecast at location $s$ at time $t+1$, then the posterior distribution for $R(s, t+1)$ is achieved by making an integral with regards to the joint posterior distribution as

$$
\begin{aligned}
P D(R(s, t+1) \mid R(s, t))= & \int P D\left(R(s, t+1) \mid \hat{R}(s, t+1), \sigma_{\epsilon}^{2}, R(s, t)\right) \\
& P D(\hat{R}(s, t+1) \mid \theta) P D(\theta \mid R(s, t)) d \hat{R}(s, t+1) d \theta
\end{aligned}
$$

where $P D$ means the posterior distribution and $\theta=\left(\beta, \sigma_{\eta}^{2}, \phi\right)^{\prime}$. According to Equation (2), we obtain

$$
R(s, t) \sim N\left(\hat{R}(s, t), \sigma_{\epsilon}^{2}\right)
$$


ALGORITHM 1: MCMC method for posterior forecasting distribution at location $s$ and time $t+1$

(1) Initialize: $\theta^{(0)}=\left(\beta^{(0)},{\sigma_{\eta}^{2(0)}}^{(0)} \phi^{(0)}, \sigma_{\epsilon}^{2(0)}\right) ; R^{(0)}=R(s, t)$

(2) Input: Observed response variable data- $R(s, t)$; Initial Values $-\theta^{(0)}$

(3) for iteration $j=1,2, \ldots, 5000$ do:

(a) Draw sample $\theta^{(j)} ; j \geq 1$ off the full conditional posterior distribution- $p\left(\theta \mid R^{(j-1)}\right)$

(b) Draw $\eta^{(j)}(s, t+1)$ from $N\left(0, \sigma_{\eta}^{2} \kappa\left(s_{i}, s_{j} ; \phi\right)\right) i, j=1, \ldots, n$;

(c) Calculate: $\hat{R}^{(j)}\left(s_{i}, t+1\right)=X\left(s_{i}, t+1\right) \beta^{(j)}+\eta^{(j)}\left(s_{i}, t+1\right)$

(d) Draw $R^{(j)}\left(s_{i}, t+1\right)$ from Normal Distribution $N\left(\hat{R}^{(j)}\left(s_{i}, t+1\right), \sigma_{\epsilon}^{2(j)}\right)$

(4) end for

(5) Output- $P(R(s, t+1) \mid R)$

So, in short, for forecasting at the next time-step:

$$
\begin{gathered}
R\left(s_{i}, t+1\right)=\hat{R}\left(s_{i}, t+1\right)+\epsilon\left(s_{i}, t+1\right), \\
\hat{R}\left(s_{i}, t+1\right)=X\left(s_{i}, t+1\right) \beta+\eta\left(s_{i}, t+1\right) .
\end{gathered}
$$

Since finding the exact posterior distribution of $R\left(s_{i}, t+1\right)$ when we use the hierarchical structure is too hard (or even impossible), a Markov Chain Monte Carlo (MCMC) method is used [Bakar 2012]. Using Gibbs sampling as a MCMC method, first we take sample $\theta^{(j)}, j \geq 1$, from the full conditional posterior distributions. After that, we take samples from the forecast distribution. For that, we draw sample $\eta^{(j)}(s, t+1)$ from $N\left(0, \sigma_{\eta}^{2} \kappa\left(s_{i}, s_{j} ; \phi\right)\right) i, j=1, \ldots, n$; at each iteration. Then, $R^{(j)}\left(s_{i}, T+1\right)$ is drawn from the normal distribution $N\left(\hat{R}^{(j)}\left(s_{i}, t+1\right), \sigma_{\epsilon}^{2(j)}\right)$, where $j \geq 1$, and we know that $\hat{R}^{(j)}\left(s_{i}, t+1\right)=X\left(s_{i}, t+1\right) \beta^{(j)}+\eta^{(j)}\left(s_{i}, t+1\right)$.

With the same logic mentioned above, if we want to predict the electric load at location $s^{\prime}$ and at time $t$, then the posterior distribution for $O\left(s^{\prime}, t\right)$ is achieved by:

$$
R\left(s^{\prime}, t\right) \sim N\left(\hat{R}\left(s^{\prime}, t\right), \sigma_{\epsilon}^{2}\right),
$$

where $\hat{R}\left(s^{\prime}, t\right)$ is the mean process of the model at site $s^{\prime}$, and we take the samples for $\hat{R}\left(s^{\prime}, t\right)$ from

$$
\left(\begin{array}{c}
\hat{R}\left(s^{\prime}, t\right) \\
\hat{R}(s, t)
\end{array}\right) \sim N\left[\left(\begin{array}{c}
X\left(s^{\prime}, t\right) \beta \\
X(s, t) \beta
\end{array}\right), \sigma_{\eta}^{2}\left(\begin{array}{cc}
1 & S_{\eta, 12} \\
S_{\eta, 21} & S_{\eta}
\end{array}\right)\right],
$$

Here, $S_{\eta, 12}=S_{\eta, 21}^{\prime}$ and $i_{t} h$ entry of $S_{\eta, 12}$ is given by $\kappa\left(s_{i}, s_{j} ; \phi\right)$. In short, we take sample $\theta^{(j)}, j \geq 1$, from off the full conditional posterior distributions and draw $\hat{R}^{(j)}\left(S^{\prime}, t\right)$ from Equation (15) and finally draw $R^{(j)}\left(s^{\prime}, t\right)$ from Equation (14).

\subsection{Prior and Posterior Distributions}

There are different methods for MCMC such as Metropolis-Hastings algorithm and Gibbs sampling. Here we use Gibbs sampling for posterior inference. We run the Gibbs sampling for 5,000 runs, and we see that it converges after 1,000 iterations. Also, we choose the first 1,000 iterations as burn-in [Gelman et al. 2004].

Every Bayesian analysis needs assigning the prior information to all the unknown parameters. So, we need to choose distributions for all the unknown parameters in our spatiotemporal model. Here, all the parameters delineating the mean are given normal prior distributions. The prior distribution for precision (inverse of the variance) parameter is given by an independent gamma prior distribution with mean 2 and variance 2 to have a good prior distribution for the variance parameter. Also, we give a flat prior Gaussian distribution with mean 0 and variance of $10^{4}$ for 
the regression coefficient of $\beta$. According to Bakar [2012], the small changes in the hyper-prior parameters yields no change in the final results, and we can say that these prior assumptions will be corrected with the training of data accumulation.

\subsection{Forecasting Performance Indices}

For validation of model performance, relative mean separation (rMSEP) is used for traffic load forecasting. Also, for electric load forecasting, symmetric mean absolute percentage error (SMAPE) is used.

Relative Mean Square Error of Prediction (rMSEP) or relative mean separation is a well-known measure for assessing the forecast performances and the precision of the method and is used in lots of research including the spatiotemporal process models such as the Bayesian Spatiotemporal Gaussian Process (BSGP) model. The rMSEP measures the expected squared distance between what the predictor predicts for a specific value and true value, relative to the distance between the true values and mean of the predictions. It measures how good the model prediction is.

In addition, Symmetric Mean Absolute Percentage Error (SMAPE) is a frequently used error metric for time series forecasting by comparing the forecast performances between different datasets. SMAPE is a measure of performance closeness based on the percentage error that has the advantage of being unit-free. Moreover, in contrast to the Mean Absolute Percentage Error (MAPE), SMAPE has both a lower bound and an upper bound and can assume a value between $0 \%$ and $100 \%$, making it easier to interpret the comparison results.

In addition, we have added the Root Mean Square Error (RMSE), another common performance indicator in the comparison.

These criteria are defined as follows:

$$
\begin{gathered}
r M S E P=\sum_{i=1}^{m}\left(\hat{p_{i}}-p_{i}\right)^{2} \mid \sum_{i=1}^{m}\left(\overline{p_{p}}-p_{i}\right)^{2}, \\
\text { SMAPE }=\frac{100 \%}{m} \sum_{i=1}^{m} \frac{\left|\hat{p_{i}}-p_{i}\right|}{\left|p_{i}\right|+\left|\hat{p_{i}}\right|}, \\
R M S E=\sqrt{\sum_{i=1}^{m}\left(\hat{p_{i}}-p_{i}\right)^{2}},
\end{gathered}
$$

where $m$ is the number of observations, $p_{i}$ is our observed data indexed by $i, \hat{p_{i}}$ is our prediction value, $\bar{p}$ is the arithmetic mean of our observation, and $\overline{p_{p}}$ is the arithmetic mean of our predictions.

\subsection{Model Validation}

For the load forecasting validation purpose, four state-of-the-art forecasting methods are chosen, which are as follows: Autoregressive Integrated Moving Average with Explanatory Variable (ARIMAX), Multi-variate Linear Regression, Support Vector Regression, and Neural Network Regression.

2.4.1 Autoregressive Integrated Moving Average with Explanatory Variable (ARIMAX) [Nguyen and Hansen 2017]. Autoregressive Integrated Moving Average with Explanatory Variable (ARIMAX) is an extension of ARIMA models when we want to consider some exogenous variables $X$ to our model. ARIMAX $(p, D, q)$ for desired time-series data $R_{t}$ and some explanatory variable $X_{t}$ can be written as

$$
\begin{aligned}
\Delta^{D} R_{t}= & \sum_{i=1}^{p} \phi_{i} \Delta^{D} R_{t-i}+\sum_{j=1}^{q} \theta_{j} \epsilon_{t-j}+\sum_{m=1}^{M} \beta_{m} X_{m, t}+\epsilon_{t} \\
& \epsilon_{t} \sim N\left(0, \sigma^{2}\right)
\end{aligned}
$$


where $\Delta^{D} R_{t}$ denotes a $D_{t h}$ differenced time series, $p$ is the autoregressive lags, $D$ is the degree of differencing, and $q$ is the number of moving average (MA) lags.

2.4.2 Multi-variate Linear Regression [Kumar et al. 2016]. Multi-variate Linear Regression (MVLR) is a time-series multi-variate linear model, which includes the two previous observations (that we obtained from ACF and PACF) and other explanatory variables. The general model of multi-variate linear regression can be driven by

$$
R_{t}=\beta_{0}+\sum_{i=1}^{2} \beta_{i} R_{t-i}+\sum_{j=1}^{m} \gamma_{j} X_{j, t}+\epsilon_{t}
$$

where $R_{t-1}$ and $R_{t-2}$ are two time series with one and two lag in time step and $X_{j, t}$ is different exogenous time series. Also, $\beta_{i}$ and $\gamma_{j}$ are coefficient of our variables and $\epsilon_{t}$ is the error term.

2.4.3 Support Vector Regression [Ceperic et al. 2013]. Support Vector Regression (SVR) is the same as Support Vector Machine (SVM) in that hinge loss and L2 regularization are used in an empirical risk minimization framework. The main goal here is to minimize the error for identifying the hyperplane that maximizes the margin and the error part is tolerated. In SVR, we usually map the input space into a higher dimensional feature space to be able to separate the observations linearly. In this research, we use the below form for support vector regression,

$$
R_{t}=\sum_{i=1}^{2} \beta_{i} R_{t-i}+\sum_{j=1}^{m} \gamma_{j} X_{j, t}+\epsilon_{t} .
$$

2.4.4 Neural Network Regression [Chow and Leung 1996]. Neural Networks (NN) is used so much recently for different tasks such as pattern recognition, classification, regression, and optimization. The performance of $\mathrm{NN}$ is mainly related to the training data and initial values for the hidden layers, the neurons value in each hidden layer and a numeric value identifying the threshold for the partial derivatives of error function as stopping criteria. For this article, we used a multilayer-perceptron approach (backpropagation) with three neurons in the hidden layer. The equation that is used here is the same as Equation (21).

\subsection{Model Generalization}

The proposed hierarchical Bayesian model based on Trend + Gaussian Process has a wide application in data modeling for the prediction of spatiotemporal variables. Therefore, the proposed model can be generalized to other state prediction problems for cyber-physical systems when the state variables exhibit strong spatial and/or temporal patterns. In cyber-physical systems for smart cities, the model can be applied to the prediction of utility consumption and fault detection in the power system as well as forecasting of traffic load, the ridership on a railway line, or the number of passengers visiting an airport in the transportation systems.

For generalization, the model should be tailored to the different natures of variable to be predicted (response variable). The model modification usually considers

- Response variable adjustment: When predicting categorical variable (e.g., power system fault classification), a logistic representation of the likelihood of certain failure mode against other types of failure (log odds) can be chosen as the response.

- Model format selection: More complex models such as higher-order polynomial and time lag can be included in the Trend. In addition, different correlation functions can be developed for Gaussian Process. 


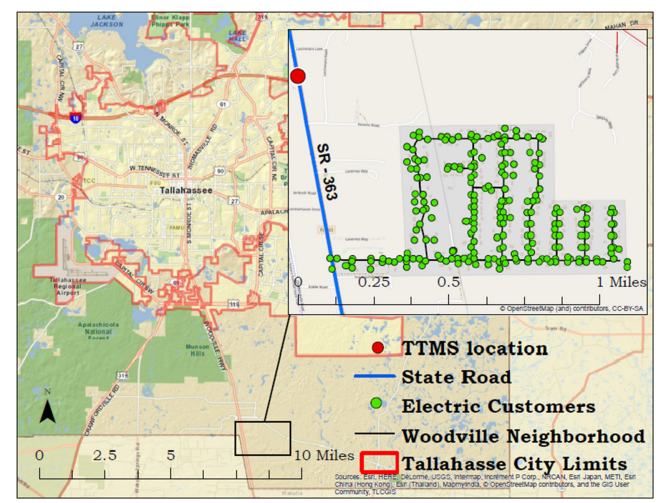

Fig. 2. Woodville in Southeast of Tallahassee.

\section{CASE STUDY}

The case study in this article is the city of Tallahassee. According to the United States Census Bureau, the city of Tallahassee, the capital of Florida, is the most populated city in Leon County, population 180,741 [5]. Florida Department of Transportation (FDOT) receives high-volume data from six different Telemetered Traffic Monitoring Sites (TTMS) storing traffic load in each direction. Also, the City of Tallahassee collects traffic load data on local roadways only for specific locations and days. However, the electricity consumption data are raw power reading $(\mathrm{kWh})$ data for every half an hour for different customer IDs that were remotely measured and stored by Meter Data Management System (MDMS) in the City of Tallahassee. Therefore, not only the traffic data but also the electricity consumption data are real world and were collected by the FDOT and the city of Tallahassee.

In addition to traffic and electricity load data, the weather data, including temperature, humidity, and solar radiation, are used and obtained from Weatherstem [39]. In this article, two case studies, two different communities in the city of Tallahassee, are chosen. For the sake of data anonymity and privacy protection, in this article, the two selected communities in Tallahassee are referred to as the Southeast community and Northeast community.

\subsection{Southeast Community}

The Southeast community has one TTMS that collects data on the Woodville highway, which connects the community to the downtown of Tallahassee. The residents of the Southeast community use the Woodville highway every day for commuting between their houses to the downtown of Tallahassee, where they work. For the first case study, this neighborhood, with 210 houses, is selected. The location of these houses is shown in Figure 2.

\subsection{Northeast Community}

In contrast to the Southeast community, the Northeast community has three TTMS locations. According to these locations, the Northeast community is divided into three different regions geographically, as shown in Figure 3. These three traffic data collection points determine the total number of vehicles coming in and out of the community. Also, the Northeast community has over 300 households with approximately 6 million electricity consumption readings studied. This community is used for traffic load forecasting due to three different locations used for traffic load data. 


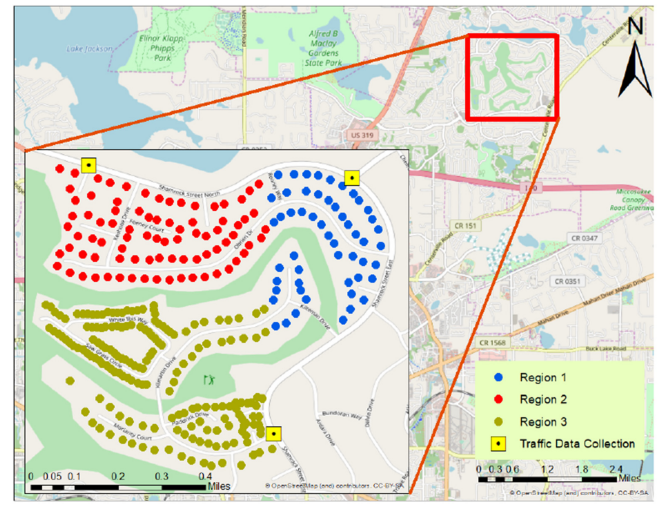

Fig. 3. Killearn in Northeast of Tallahassee.

Table 1. Summary of Training and Testing Data in Different Scenarios

\begin{tabular}{|c|c|c|c|}
\hline \multicolumn{4}{|c|}{ Traffic Load Forecasting } \\
\hline Community & Scebarios & $\begin{array}{l}\text { Number of Training } \\
\text { Observations }\end{array}$ & Number of Testing Observations \\
\hline \multirow{3}{*}{$\begin{array}{l}\text { Southeast } \\
\text { Community }\end{array}$} & $\begin{array}{l}\text { Northbound AM } \\
\text { Peak Traffic }\end{array}$ & $\begin{array}{l}17 \text { days } \times 7 \text { half an } \\
\text { hour } \times 4 \text { geographical } \\
\text { regions }=\mathbf{4 7 6}\end{array}$ & $\begin{array}{l}-1 \text { day } \times 7 \text { half an hour } \times 4 \text { geographical regions }=\mathbf{2 8} \\
-3 \text { days } \times 7 \text { half an hour } \times 4 \text { geographical regions }=\mathbf{8 4} \\
-7 \text { days } \times 7 \text { half an hour } \times 4 \text { geographical regions }=\mathbf{1 9 6}\end{array}$ \\
\hline & $\begin{array}{l}\text { Southbound PM } \\
\text { Peak Traffic }\end{array}$ & $\begin{array}{l}17 \text { days } \times 7 \text { half an } \\
\text { hour } \times 4 \text { geographical } \\
\text { regions }=\mathbf{4 7 6}\end{array}$ & $\begin{array}{l}-1 \text { day } \times 7 \text { half an hour } \times 4 \text { geographical regions }=\mathbf{2 8} \\
-3 \text { days } \times 7 \text { half an hour } \times 4 \text { geographical regions }=\mathbf{8 4} \\
-7 \text { days } \times 7 \text { half an hour } \times 4 \text { geographical regions }=\mathbf{1 9 6}\end{array}$ \\
\hline & $\begin{array}{l}\text { Daily Northbound } \\
\text { and Southbound } \\
\text { Traffic }\end{array}$ & $\begin{array}{l}17 \text { days } \times 48 \text { half an } \\
\text { hour } \times 4 \text { geographical } \\
\text { regions }=\mathbf{3 2 6 4}\end{array}$ & $\begin{array}{l}-1 \text { day } \times 48 \text { half an hour } \times 4 \text { geographical regions }=\mathbf{1 9 2} \\
-3 \text { days } \times 48 \text { half an hour } \times 4 \text { geographical regions }=\mathbf{5 7 6} \\
-7 \text { days } \times 48 \text { half an hour } \times 4 \text { geographical regions }=\mathbf{1 3 4 4}\end{array}$ \\
\hline \multicolumn{2}{|c|}{ Northeast Community } & $\begin{array}{l}11 \text { days } \times 48 \text { half an } \\
\text { hour } \times 3 \text { geographical } \\
\text { regions }=\mathbf{1 5 8 4}\end{array}$ & $\begin{array}{l}-1 \text { day } \times 48 \text { half an hour } \times 3 \text { geographical regions }=\mathbf{1 4 4} \\
-3 \text { days } \times 48 \text { half an hour } \times 3 \text { geographical regions }=\mathbf{4 3 2} \\
-7 \text { days } \times 48 \text { half an hour } \times 3 \text { geographical regions }=\mathbf{1 0 0 8}\end{array}$ \\
\hline \multicolumn{4}{|c|}{ Electric Load Forecasting } \\
\hline \multicolumn{3}{|c|}{ Number of Training Observations } & Number of Testing Observations \\
\hline \multicolumn{3}{|c|}{14 days $\times 48$ half an hour $\times 145$ Houses $=\mathbf{9 7 4 4 0}$} & $\begin{array}{l}-1 \text { day } \times 48 \text { half an hour } \times 145 \text { Houses }=\mathbf{6 9 6 0} \\
-3 \text { days } \times 48 \text { half an hour } \times 145 \text { Houses }=\mathbf{2 0 8 8 0} \\
-7 \text { days } \times 48 \text { half an hour } \times 145 \text { Houses }=\mathbf{4 8 7 2 0}\end{array}$ \\
\hline
\end{tabular}

\section{RESULTS AND DISCUSSIONS}

In this section, results are divided into two main subsections: traffic load forecasting and electric load forecasting. In each subsection, results obtained from the proposed BSGP and state-of-the-art methods are presented and compared to each other. In this article, most of the statistical analyses were implemented in the R programming language using different packages such as the coda and spTimer packages [Bakar and Sahu 2015a, 2015b]. The training and testing data that we used are shown in Table 1.

\subsection{Traffic Load Forecasting}

In this subsection, results obtained from the BSGP and state-of-the-art methods for the Southeast and Northeast communities are represented in two different subsections as follows: 

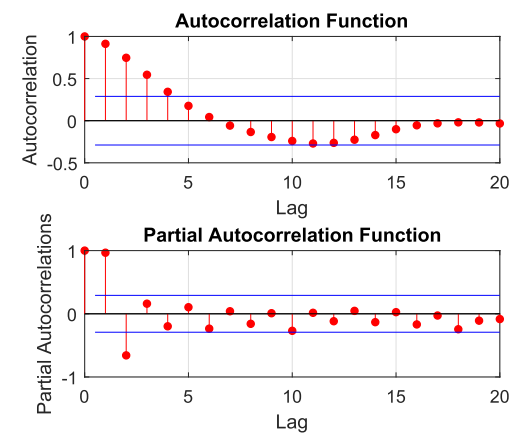

Fig. 4. Autocorrelation (ACF) and Partial Autocorrelation (PACF) for stationarized Traffic load time series for Northbound traffic.
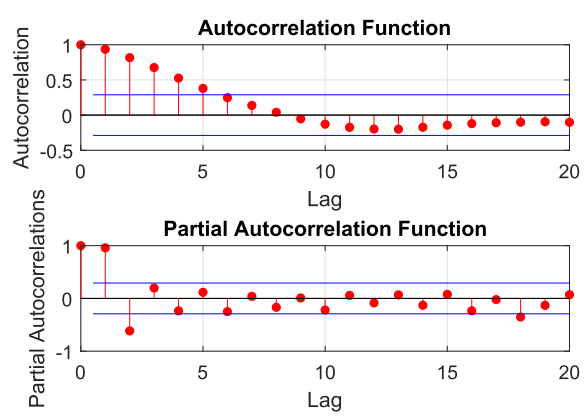

Fig. 5. Autocorrelation (ACF) and Partial Autocorrelation (PACF) for stationarized Traffic load time series for Southbound traffic.

4.1.1 Southeast Community. As explained in the previous section, there are 210 houses in the Southeast community. For this case study, the Southeast community is divided into four different regions geographically: Northeast, Northwest, Southeast, Southwest. Each region has a number of houses with smart meters to measure electrical consumption. In this case study, we use the total electricity consumption in the region, which is the sum of electrical consumption of each household in that region.

As explained in the methodology, first we need to determine the $p$ in the Trend. So the $p$ time lag is obtained by observing the ACF and PACF figures. Before that, according to the Augmented Dickey-Fuller (ADF) method, the traffic load time series for the Southeast community is stationary (the $p$-value of the ADF almost 0.01). After finding that the traffic load time series is stationary, then we can obtain the number of autocorrelation terms based on the ACF and PACF to have them in the model.

According to Figures 4 and 5 regarding traffic load time series in the Northbound and Southbound directions in the Southeast community, they show that the ACF has a smooth declining pattern, which confirms the results obtained from the Augmented Dickey-Fuller (ADF) test that said the Northbound and Southbound traffic time series are stationary. Moreover, by looking at the PACF figures, it is clear that the PACF in Figures 4 and 5 cut off at lag "2," which suggests that two previous observations, $R_{t-1}$ and $R_{t-2}$, will be enough to have in the model at a $5 \%$ significant level. These two terms bring sufficient information for the traffic load forecasting in the Northbound and Southbound directions at 5\% significant level.

For selecting the model order, all the training data for each case study and each scenario are used. For example, for traffic load forecasting in the Southeast community in the Northbound AM Peak, all 476 observations from the traffic load variable are used to plot the ACF and PACF figures and determine the number of autocorrelations in traffic load time series.

Figure 6 shows a 1-day traffic load through the Southbound and Northbound directions. As observed in Figure 6, the traffic peak for the Northbound direction occurs in the morning when people are leaving their house and going to work (to downtown Tallahassee). However, the Southbound traffic peak occurs in the afternoon when people are coming back to their houses from work. With an understanding of the fact that these AM and PM traffic peaks are important in transportation and traffic forecasting, we will define three scenarios for forecasting: (a) forecasting the Northbound direction traffic load on a weekday (February 2, 2015) in the morning (6AM-9AM) using Northbound direction traffic data in the mornings (6AM-9AM) of weekdays in January 2015, 


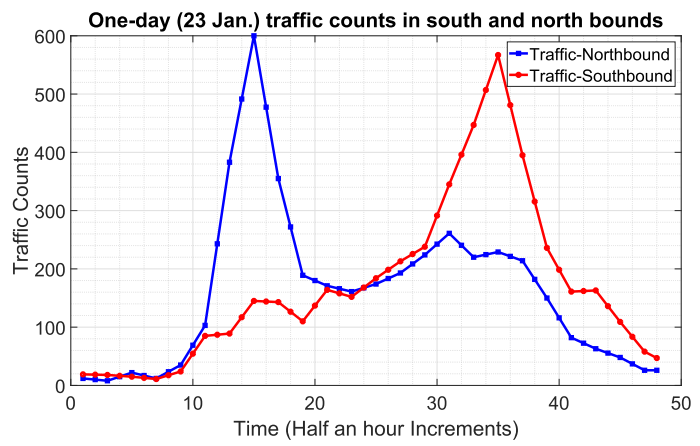

Fig. 6. One-day traffic counts in the Northbound and Southbound directions for the Woodville neighborhood.

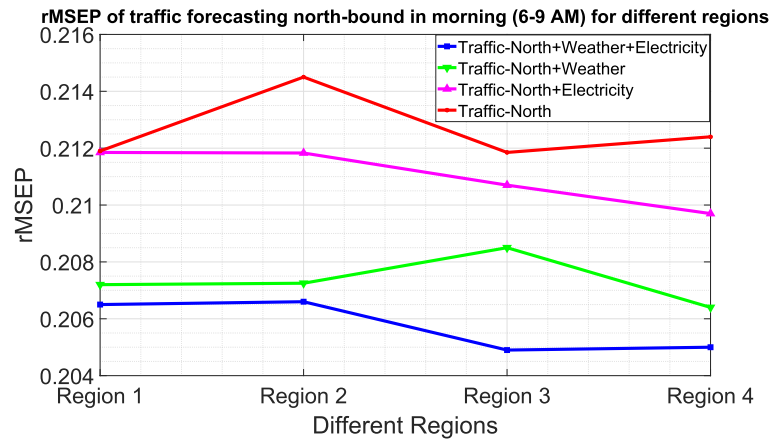

Fig. 7. rMSEP of Northbound AM Peak traffic forecasting for four regions of the Southeast community.

(b) forecasting the Southbound direction traffic load on a weekday (February 2, 2015) in the afternoon (4PM-7PM) using Southbound direction traffic data in the afternoons (4PM-7PM) of weekdays in January 2015, and (c) forecasting the traffic load for both directions on a weekday (February $2,2015)$ for the whole day using the traffic data in both directions in the whole weekdays of January 2015. The weekdays of January are as follows: 1, 5, 6, 7, 8, 12, 13, 14, 15, 19, 20, 21, 22, 26, 27, 28 , and 29.

\section{(a) Northbound AM Peak Traffic Forecasting}

Since the morning (AM) traffic peak occurs in the Northbound direction when the people are going to work, forecasting the Northbound AM traffic (6AM-9AM) appears to be more meaningful. In Figure 7, the rMSEP values obtained from our model for all four different regions are shown, and the lowest rMSEP belongs to the scenario by having all the parameters in the model. This figure shows that the forecasting rMSEP will be decreased by $3.2 \%$ as a result of the data fusion where electricity consumption and weather information are fed to forecast the traffic.

\section{(b) Southbound PM Peak Traffic Forecasting}

In this section, we present the results of traffic load forecasting for the PM peak (4PM-7PM) Southbound direction when the people are coming back home from work. Figure 8 shows the rMSEP of traffic forecasting obtained from our method for the four different regions. As shown in the figure, the lowest rMSEP belongs to the scenario with having all the transportation, weather, and electricity data. As a result, with the help of data fusion, we could decrease the rMSEP by $31.8 \%$. Also, detailed expression of traffic forecasting error for 1-day ahead and for different prediction horizons are shown in Tables 4 and 5 respectively. 


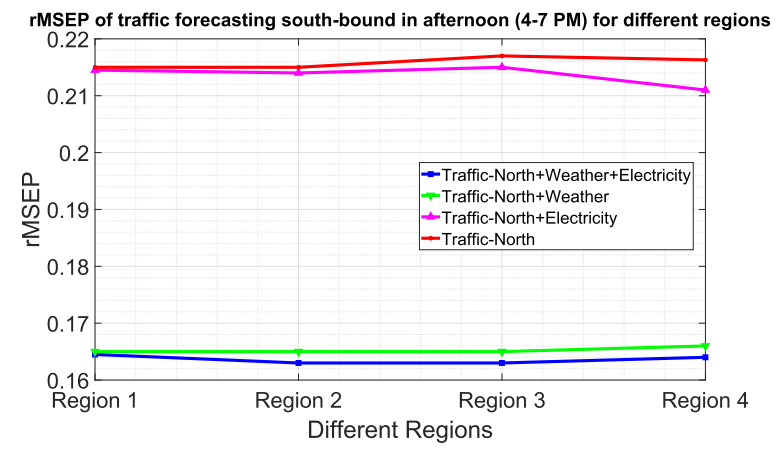

Fig. 8. rMSEP of Southbound PM Peak traffic forecasting for four regions of the Southeast community.

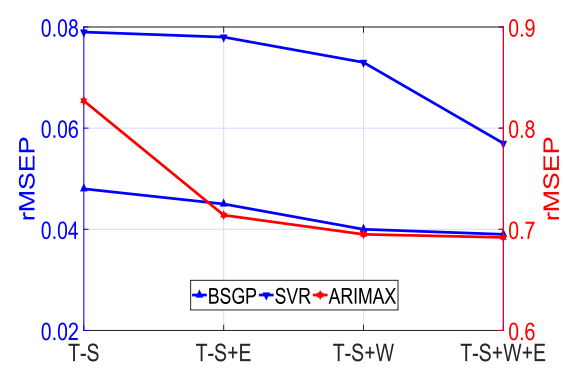

Fig. 9. rMSEP of daily forecasting in the Southbound direction for four different scenarios.

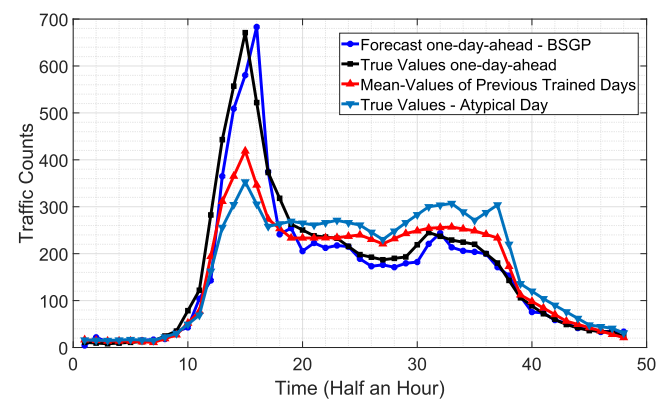

Fig. 10. Forecast traffic load profile and its true values for a weekday in the Southeast community along with mean values of previous traffic and traffic load of an atypical day.

\section{(c) Daily Northbound and Southbound Forecasting}

The third scenario is forecasting the traffic load in both the North and Southbound directions for a complete day. Figure 9 shows the rMSEP of traffic forecasting obtained from our method in the Southbound direction for the four scenarios in the Southeast community. By adding the electricity and weather information to the model, the error is decreased. The lowest rMSEP belongs to the scenario with having Southbound traffic, weather, and electricity information (rMSEP $=0.39$ ).

The highest rMSEP belongs to the Northbound-traffic-only case. The rMSEP figure is emphasizing that the forecasting error is decreased and the forecasting accuracy is increased as a result of data fusion and the addition of more information from other data resources. Although the impact of weather is usually more than the impact of electricity, in the Northbound direction, the impact of adding electricity becomes higher than that of the weather. The lowest RMSE belongs to the Southbound traffic, with all the weather and electricity data added to the model. The highest RMSE, ohowever, belongs to the case without the addition of weather and electricity data. Moreover, Figure 10 shows the forecast traffic load profile and true values for a weekday in the Southeast community. It also shows the mean values of the previous traffic load a long with an atypical day. This figure clearly shows that the proposed BSGP can capture the traffic load pattern well even for the peak of the traffic that is higher than the mean values of previous traffic loads.

\section{Comparison}

For validation of the proposed hierarchical Bayesian method, we compared its performance with two widely used methods for forecasting: Autoregressive Integrated Moving Average with 
Table 2. Error Table for 1-Day Northbound Traffic Forecasting in AM (T-N:

Traffic Northbound, W: Weather, E: Electricity)-(BSGP: Bayesian

Spatiotemporal Gaussian Process, ARIMAX: Autoregressive Integrated

Moving Average with Explanatory Variable, SVR: Support Vector

Regression)-(Numbers in Parentheses Show the Percentage of Decrease in

Errors Compared to the First Scenario with Traffic Data Only)

\begin{tabular}{|l|l||l|c|c|l|}
\hline Error & Methods & T-N & T-N +E & T-N +W & T-N +W +E \\
\hline \hline \multirow{3}{*}{ rMSEP } & BSGP & 0.213 & 0.211 & 0.207 & $0.206(-3.2)$ \\
\cline { 2 - 6 } & ARIMAX & 1.562 & 0.412 & 0.385 & $0.379(-75.8)$ \\
\cline { 2 - 6 } & SVR & 0.348 & 0.317 & 0.313 & $0.284(-18.2)$ \\
\hline \multirow{3}{*}{ RMSE } & BSGP & 50.84 & 50.73 & 49.16 & $49.07(-3.5)$ \\
\cline { 2 - 6 } & ARIMAX & 187.34 & 78.47 & 73.29 & $72.06(-61.5)$ \\
\cline { 2 - 6 } & SVR & 67.98 & 61.92 & 61.08 & $55.42(-18.5)$ \\
\hline
\end{tabular}

Table 3. Error Table for Different Prediction Horizons for Northbound Traffic

Forecasting in AM When All Input Variables Are Included $(\mathrm{T}-\mathrm{N}+\mathrm{W}+\mathrm{E})$

\begin{tabular}{|l||l|l|l|l||l|l|l|}
\hline rMSEP & 1 day & 3 days & 7 days & RMSE & 1 day & 3 days & 7 days \\
\hline \hline BSGP & 0.206 & 0.218 & 0.192 & BSGP & 49.07 & 58.95 & 56.68 \\
\hline ARIMAX & 0.379 & 0.652 & 0.524 & ARIMAX & 72.06 & 110.02 & 105.60 \\
\hline SVR & 0.284 & 0.328 & 0.291 & SVR & 55.42 & 72.11 & 69.61 \\
\hline
\end{tabular}

Explanatory Variable (ARIMAX) and Support Vector Regression (SVR). Moreover, model order selection for comparison method is performed under the same conditions as the proposed method. For the MVLR, SVR, and NN, all the training data for each case study and each scenario is used. Also, all the methods were tuned, and the best parameters were selected for them. For ARIMAX, the best model was selected by searching for possible models in the auto function in the forecast package in the $R$ programming language, which returns the best ARIMA model according to the Bayesian Information Criterion (BIC). For the SVR, the radial basis function kernel was used, and the best parameters were obtained after parameter tuning with 10 -fold cross-validation, which is an effective way of reducing the overfitting problem. For NN, a multilayer perceptron approach (backpropagation) was used, and the best initial values were determined after cross-validation and parameter tuning for the hidden layers, the number of neurons in each hidden layer, and the threshold for the partial derivatives of the error function as stopping criteria. The best NN, with three neurons in a hidden layer, a threshold equal to 0.01 , and $1 \times 10^{7}$ as the maximum number of steps, is chosen.

The detailed results obtained from the proposed method, ARIMAX and SVR, are shown in Tables 2, 4, and 6. As is clear, the Bayesian Spatiotemporal Gaussian Process (BSGP) method can decrease the rMSEP by $31.8 \%$. The least reduction in rMSEP occurs in forecasting the NorthboundAM peak by $3.3 \%$ and $3.2 \%$, respectively. According to all the methods discussed, by considering weather and electricity information to the model, the error is decreased. This indicates that data fusion and having more information from different urban infrastructures such as weather and electricity consumption can help to forecast the traffic volume more precisely.

Results show that, according to all the methods, by having electricity information, the traffic forecasting error (rMSEP) will be decreased. The best scenario is the one that has both weather and electricity information included. As mentioned, the highest decrease in error by data fusion occurs in Southbound traffic. This may be explained by human behavior. That is, people who do 
Table 4. Error Table for 1-Day Southbound Traffic Forecasting in PM (T-S:

Traffic Southbound, W: Weather, E: Electricity)-(BSGP: Bayesian Spatiotemporal Gaussian Process, ARIMAX: Autoregressive Integrated

Moving Average with Explanatory Variable, SVR: Support Vector Regression)-(Numbers in Parenthesis Show the Percentage of Decrease in Errors Compared to the First Scenario with Traffic Data Only)

\begin{tabular}{|l|l||l|c|c|c|}
\hline Error & Methods & T-S & T-S +E & T-S +W & T-S +W +E \\
\hline \hline \multirow{3}{*}{ rMSEP } & BSGP & 0.215 & 0.213 & 0.165 & $0.163(-31.8)$ \\
\cline { 2 - 6 } & ARIMAX & 0.159 & 0.093 & 0.082 & $0.079(-50.3)$ \\
\cline { 2 - 6 } & SVR & 0.233 & 0.198 & 0.193 & $0.171(-26.4)$ \\
\hline \multirow{3}{*}{ RMSE } & BSGP & 70.58 & 70.31 & 60.78 & $50.74(-28.1)$ \\
\cline { 2 - 6 } & ARIMAX & 117.92 & 68.96 & 60.54 & $59.23(-49.7)$ \\
\cline { 2 - 6 } & SVR & 94.65 & 80.42 & 78.33 & $65.32(-30.9)$ \\
\hline
\end{tabular}

Table 5. Error Table for Different Prediction Horizons for Southbound Traffic Forecasting in PM When All Input Variables Are Included (T-S+W+E)

\begin{tabular}{|l||l|l|l|l||l|l|l|}
\hline rMSEP & 1 day & 3 days & 7 days & RMSE & 1 day & 3 days & 7 days \\
\hline \hline BSGP & 0.163 & 0.172 & 0.172 & BSGP & 50.74 & 60.76 & 58.86 \\
\hline ARIMAX & 0.079 & 0.203 & 0.283 & ARIMAX & 59.23 & 66.68 & 76.73 \\
\hline SVR & 0.171 & 0.299 & 0.268 & SVR & 65.32 & 80.09 & 73.67 \\
\hline
\end{tabular}

Table 6. Error Table for 1-Day North- and Southbound Traffic Forecasting in Whole Day (T-N: Traffic Northbound, T-S: Traffic Southbound, W: Weather, E: Electricity)-(BSGP: Bayesian Spatiotemporal Gaussian Process, ARIMAX: Autoregressive Integrated Moving Average with Explanatory Variable, SVR: Support Vector Regression)-(Numbers in Parenthesis Show the Percentage of Decrease in Errors Compared to the First Scenario with Traffic Data Only)

\begin{tabular}{|l|l||l|l|l|l|l|l|l|l|}
\hline Error & Methods & T-S & T-S +E & T-S +W & T-S +W +E & T-N & T-N +E & T-N +W & T-N +W +E \\
\hline \hline \multirow{4}{*}{ rMSEP } & BSGP & 0.048 & 0.045 & 0.040 & $0.039(-23.1)$ & 0.058 & 0.054 & 0.056 & $0.053(-9.4)$ \\
\cline { 2 - 10 } & ARIMAX & 0.827 & 0.714 & 0.695 & $0.692(-16.3)$ & 0.873 & 0.872 & 0.872 & $0.872(-0.1)$ \\
\cline { 2 - 10 } & SVR & 0.079 & 0.078 & 0.073 & $0.057(-27.7)$ & 0.084 & 0.076 & 0.074 & $0.067(-19.8)$ \\
\hline \multirow{3}{*}{ RMSE } & BSGP & 36.17 & 34.58 & 34.23 & $32.06(-11.4)$ & 34.95 & 34.90 & 34.62 & $34.32(-1.8)$ \\
\cline { 2 - 10 } & ARIMAX & 145.64 & 134.28 & 129.74 & $129.07(-11.4)$ & 139.54 & 138.89 & 138.89 & $138.89(-0.5)$ \\
\cline { 2 - 10 } & SVR & 69.93 & 69.05 & 63.65 & $49.05(-29.8)$ & 53.50 & 47.41 & 45.97 & $41.35(-22.7)$ \\
\hline
\end{tabular}

not leave their homes in the morning may be asleep, and, therefore, they may not be using electricity in the morning. As such, adding electricity data does not provide substantial information regarding the traffic load. However, those people who are in their house during PM peaks usually use electricity. Therefore, increasing electricity consumption will indicate that they are in their house, and there will be fewer cars in the highway. As such, we can state that electricity data can convey more information in the afternoon rather than in the morning. All in all, data fusion of electricity and weather information into transportation networks can increase the traffic prediction accuracy and can decrease the traffic prediction error. Also, results of whole-day traffic forecasting in Northbound and Southbound directions for different prediction horizons are presented in Tables 7 and 8 respectively. 
Table 7. Error Table for Different Prediction Horizons for Whole-Day Northbound Traffic Forecasting When All Input Variables Are Included $(\mathrm{T}-\mathrm{N}+\mathrm{W}+\mathrm{E})$

\begin{tabular}{|l||l|l|l|l||l|l|l|}
\hline rMSEP & 1 day & 3 days & 7 days & RMSE & 1 day & 3 days & 7 days \\
\hline \hline BSGP & 0.053 & 0.065 & 0.064 & BSGP & 34.32 & 37.99 & 38.37 \\
\hline ARIMAX & 0.872 & 0.988 & 1.014 & ARIMAX & 138.89 & 148.26 & 153.35 \\
\hline SVR & 0.067 & 0.079 & 0.083 & SVR & 41.35 & 51.56 & 59.36 \\
\hline
\end{tabular}

Table 8. Error Table for Different Prediction Horizons for Whole-Day Southbound Traffic Forecasting When All Input Variables Are Included (T-S+W+E)

\begin{tabular}{|l||l|l|l|l||l|l|l|}
\hline rMSEP & 1 day & 3 days & 7 days & RMSE & 1 day & 3 days & 7 days \\
\hline \hline BSGP & 0.039 & 0.043 & 0.044 & BSGP & 32.06 & 33.31 & 33.67 \\
\hline ARIMAX & 0.692 & 0.806 & 1.012 & ARIMAX & 129.07 & 143.26 & 163.58 \\
\hline SVR & 0.057 & 0.471 & 0.136 & SVR & 49.05 & 71.92 & 59.74 \\
\hline
\end{tabular}
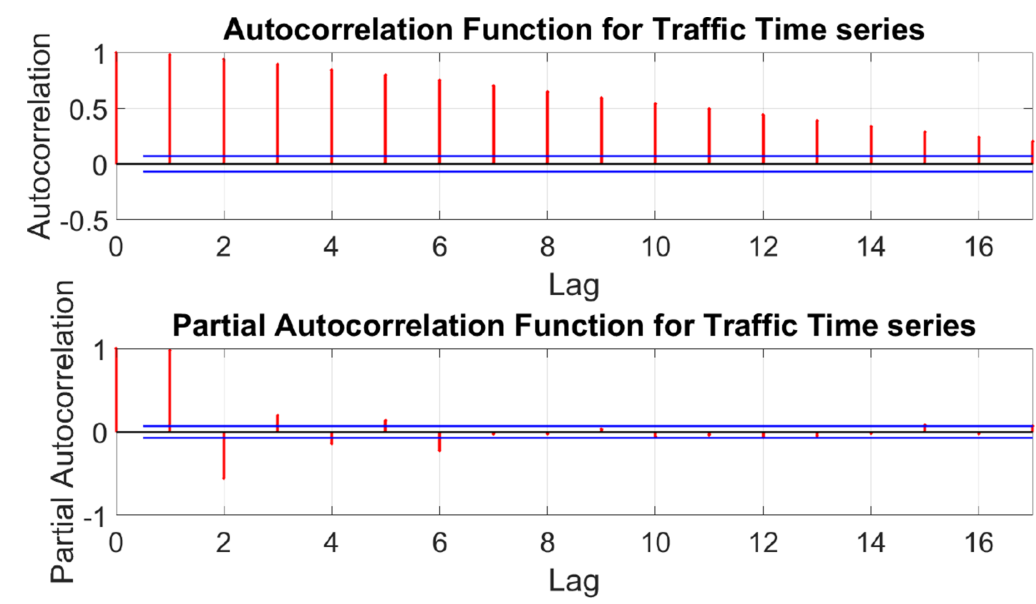

Fig. 11. Autocorrelation (ACF) and Partial Autocorrelation (PACF) for stationarized time series in out-bound traffic.

4.1.2 Northeast Community. The Northeast community has three different traffic load collection sites that determine the number of vehicles coming in and out of the community. Therefore, for each traffic load collection site, there are two traffic variables, including traffic load for those vehicles moving into the community and traffic load for those vehicles going out of the community. However, the whole community has more than 300 houses, where the sum of electricity consumption of all houses located in each region is considered as the electricity consumption variable. This community is divided into three regions geographically as shown in Figure 3.

As explained, the first step is to determine the $p$ time lag in the Trend in the BSGP model. According to Figures 11 and 12, the time series in in-bound and out-bound traffic directions are stationary due to their smooth declining patterns. Furthermore, based on the PACF figures, six and eight previous observations are chosen for traffic in the out-bound direction and the in-bound direction, respectively. 

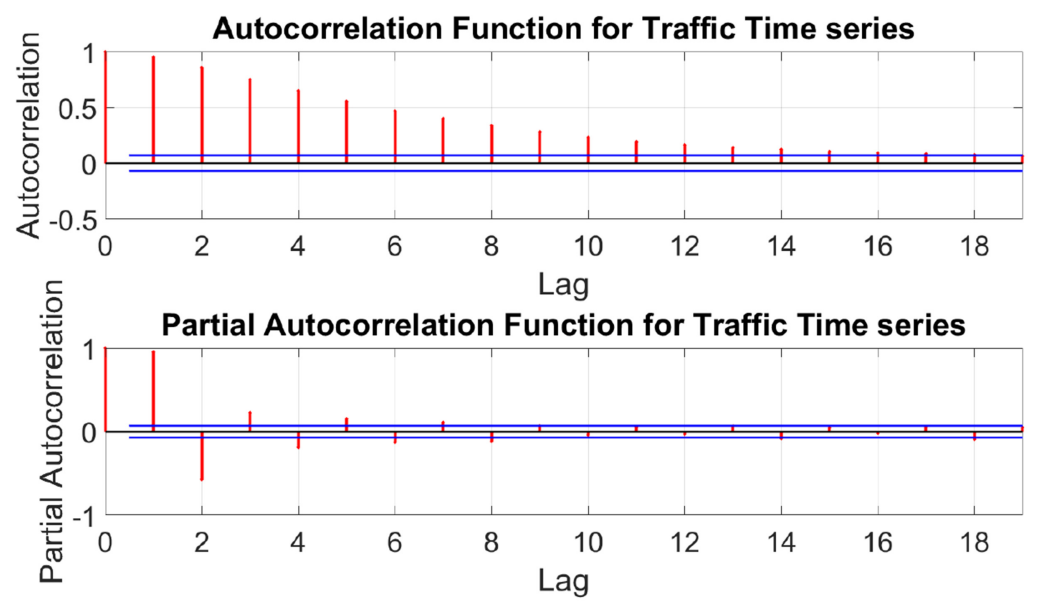

Fig. 12. Autocorrelation (ACF) and Partial Autocorrelation (PACF) for stationarized time series in in-bound traffic.

Table 9. Error Table for 1-Day Forecasting in In-bound and Out-bound Traffic in the Northeast Community (OT: Out-bound Traffic, IT: In-bound Traffic, W: Weather, E: Electricity)-(BSGP: Bayesian Spatiotemporal Gaussian Process, ARIMAX: Autoregressive Integrated Moving Average with Explanatory Variable, SVR: Support Vector Regression)-(Numbers in Parenthesis Show the Percentage of Decrease in Errors Compared to the First Scenario with Traffic Data Only)

\begin{tabular}{|l|l||l|l|l|l|l|l|l|l|}
\hline Error & Methods & IT & IT $+\mathrm{E}$ & IT $+\mathrm{W}$ & IT $+\mathrm{W}+\mathrm{E}$ & $\mathrm{OT}$ & $\mathrm{OT}+\mathrm{E}$ & $\mathrm{OT}+\mathrm{W}$ & $\mathrm{OT}+\mathrm{W}+\mathrm{E}$ \\
\hline \hline \multirow{4}{*}{ rMSEP } & BSGP & 0.202 & 0.186 & 0.184 & $0.175(-13.4)$ & 0.145 & 0.134 & 0.132 & $0.126(-13.1)$ \\
\cline { 2 - 11 } & ARIMAX & 0.985 & 0.958 & 0.950 & $0.908(-7.8)$ & 0.998 & 0.981 & 0.980 & $0.968(-3)$ \\
\cline { 2 - 10 } & SVR & 0.229 & 0.208 & 0.205 & $0.195(-14.8)$ & 0.154 & 0.136 & 0.131 & $0.129(-16.2)$ \\
\hline \multirow{3}{*}{ RMSE } & BSGP & 4.96 & 4.58 & 4.55 & $4.44(-10.5)$ & 6.45 & 6.21 & 6.20 & $6.02(-6.6)$ \\
\cline { 2 - 10 } & ARIMAX & 22.01 & 21.92 & 21.33 & $21.31(-3.2)$ & 30.61 & 30.59 & 30.11 & $29.99(-2.1)$ \\
\cline { 2 - 10 } & SVR & 14.65 & 14.29 & 13.87 & $13.04(-10.9)$ & 9.94 & 9.33 & 9.63 & $9.09(-8.6)$ \\
\hline
\end{tabular}

Table 10. Error Table for Different Prediction Horizons for Forecasting of Out-Bound Traffic When All Input Variables Are Included $(\mathrm{OT}+\mathrm{W}+\mathrm{E})$

\begin{tabular}{|l||l|l|l|l||l|l|l|}
\hline rMSEP & 1 day & 3 days & 7 days & RMSE & 1 day & 3 days & 7 days \\
\hline \hline BSGP & 0.13 & 0.14 & 0.23 & BSGP & 6.02 & 6.79 & 6.99 \\
\hline ARIMAX & 0.97 & 1.01 & 1.03 & ARIMAX & 29.99 & 32.26 & 33.65 \\
\hline SVR & 0.13 & 0.13 & 0.14 & SVR & 9.09 & 10.78 & 10.88 \\
\hline
\end{tabular}

Results obtained from the proposed method, ARIMAX, and SVR for 1-day forecasting for inbound and out-bound traffic are presented in Table 9. Both the rMSEP and RMSE for these methods are represented in different scenarios such as only traffic data, traffic with electricity, traffic with environmental variables, and traffic along with electricity and environmental variables are included in the model.

Also, the error tables of forecasting in out-bound and in-bound traffic obtained from the proposed BSGP, ARIMAX, and SVR for different prediction horizons, including 1 day, 3 days, and 7 days, are shown in Tables 10 and 11, respectively. Results indicate that by increasing the 
Table 11. Error Table for Different Prediction Horizons for Forecasting of In-bound Traffic When All Input Variables Are Included (IT+W+E)

\begin{tabular}{|l||l|l|l|l||l|l|l|}
\hline rMSEP & 1 day & 3 days & 7 days & RMSE & 1 day & 3 days & 7 days \\
\hline \hline BSGP & 0.17 & 0.19 & 0.21 & BSGP & 4.44 & 7.39 & 8.39 \\
\hline ARIMAX & 0.91 & 0.99 & 1.02 & ARIMAX & 21.33 & 38.20 & 39.71 \\
\hline SVR & 0.19 & 0.24 & 0.25 & SVR & 13.04 & 17.11 & 18.97 \\
\hline
\end{tabular}
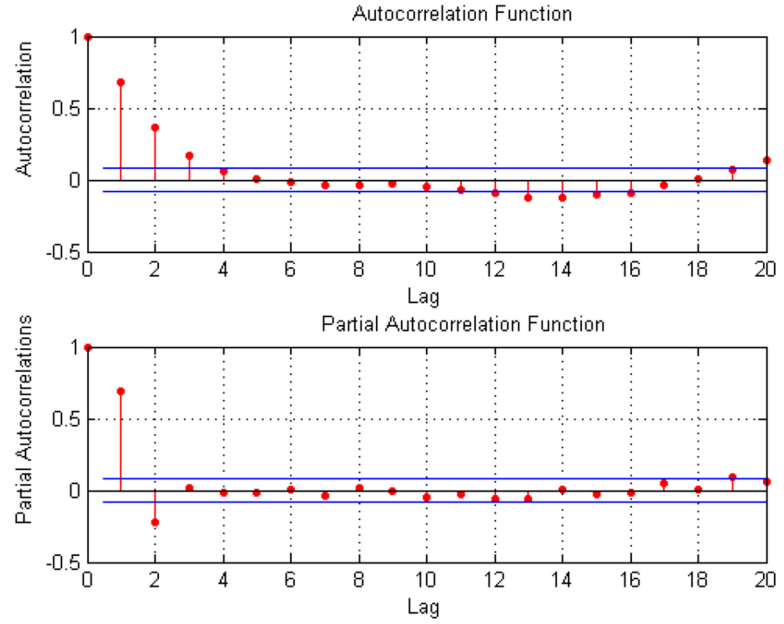

Fig. 13. Autocorrelation (ACF) and Partial Autocorrelation (PACF) for stationarized electrical load time series in the Southeast community.

prediction horizons, the error will be increased. Therefore, the smallest errors belong to 1-day forecasting and to BSGP. Both RMSE and rMSEP prove that the BSGP outperforms the other methods and increasing the prediction horizons increase the error and decrease the accuracy.

\subsection{Electric Load Forecasting}

In this subsection, the results of electric load forecasting obtained from the BSGP and state-ofthe-art methods for the Southeast community are expressed. As explained in the case study, there are data for 210 houses in the Southeast community in Tallahassee. For this article, $70 \%$ of them (145 houses) are selected randomly for training. So training data include 145 houses having electrical consumption data from January 1 until January 14. The goal is to forecast the electric load for January 15 (next day) for the trained houses.

As explained, the $p$ time lag in the Trend in the BSGP model is obtained by observing the ACF and PACF figures. Before that, based on the Augmented Dickey-Fuller (ADF), the electric load time series for the Southeast community is stationary ( $p$-value of the ADF almost 0.01 ). After finding that the time series is stationary, we can obtain the number of autocorrelation terms based on the $\mathrm{ACF}$ and PACF to have them in the model.

According to the Figure 13, the ACF figure has a smooth declining pattern that confirms the results obtained from the ADF test, which indicates that the time series is stationary. Moreover, by looking at the PACF figures, it is clear that the PACF in Figure 13 cut off at lag "2," which suggests that two previous observations, $R_{t-1}$ and $R_{t-2}$, will be enough to have in the model at a 


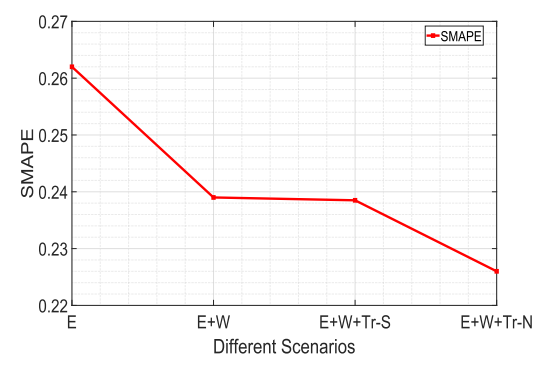

Fig. 14. SMAPE decrease in BSGP model with using multi-source data fusion framework for electric load forecasting.

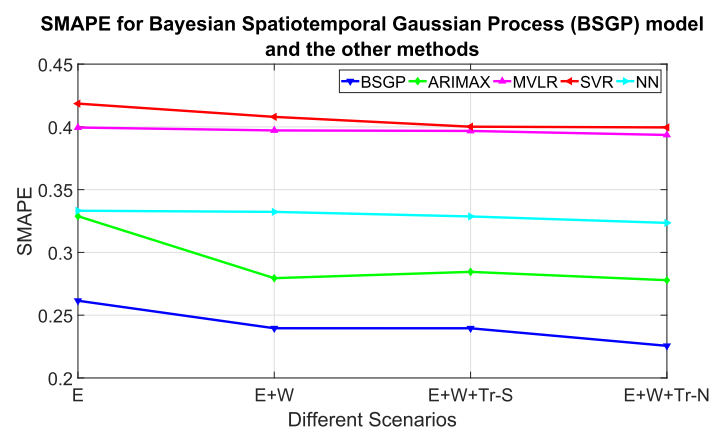

Fig. 15. SMAPE of the proposed method and the other four methods in four different scenarios.

5\% significant level. These two terms provide sufficient information for traffic load forecasting at a $5 \%$ significant level.

Based on our predictors, four scenarios are defined. In the first scenario, only the electrical consumption data are included. In the second scenario, we have the electrical data along with weather data, including temperature, humidity, and solar radiation. In the third scenario, the mobility information is added. The traffic load (the number of vehicles) toward south direction (in Woodville highway) is considered with electrical and weather data. At last, in the fourth scenario, the traffic load in the north direction with electrical and weather data are considered altogether. From now on, the "traffic load" is called "transportation" for convenience.

In Figure 14, the average SMAPE of load forecasting obtained from the proposed Bayesian spatiotemporal Gaussian process model for all houses are shown. The SMAPE is achieved from the load forecasting of January 15 day (next day). It is clear that the SMAPE is decreased when urban mobility along with weather data are considered in the model. The worst scenario is the one considering only the electricity data, and the best scenario is the one with studying the electrical data with weather and transportation-north data. Figure 14 clearly shows that meteorological information and adding urban mobility can decrease errors in the electricity load forecasting.

Also, SMAPE of load forecasting in different four scenarios for our proposed method, ARIMAX, Multi-variate Linear Regression (MVLR), Support Vector Regression (SVR), and Neural Network $(\mathrm{NN})$ are shown in Table 12 in detail. As is clear, the proposed method can decrease the SMAPE by almost $16 \%$ when the weather and Northbound transformation is added to the model.

Based on Table 12, the least SMAPE belongs to the scenario where there is electricity, weather, and transportation-north data and belongs to the proposed method that is 0.23 . A common thing between all these methods is that by considering meteorological and urban mobility information, the error will be decreased. As a result, the accuracy will be increased. Moreover, error table of electric load forecasting for different prediction horizons and all inputs is shown in Table 13.

As shown in Figure 15, by adding weather and transportation information, the error is decreased. Except for ARIMAX, the error is decreased by going from just having electricity data to a scenario with having electricity, weather, and transportation-north data. This figure clearly shows the importance of urban mobility in load forecasting, and it clearly demonstrates the efficacy of the proposed method among the others. So, based on the results obtained and shown in Tables 2, 3, 4, and 5:

- The fewest errors are mostly obtained from the proposed Bayesian Spatiotemporal Gaussian Process (BSGP) model, 
Table 12. SMAPE of Load Forecasting in Four Different Scenarios (Just Having Electricity (E), Electricity and Weather $(E+W)$, Electricity, Weather and Transportation South $(E+W+T r . S)$ and Having Electricity, Weather, and Transportation North $(\mathrm{E}+\mathrm{W}+\mathrm{Tr} . \mathrm{N})$ ) for Bayesian Spatio-temporal Gaussian Process Model (BSGP), Autoregressive Integrated Moving Average with Explanatory Variable (ARIMAX), Multi-Variate Linear Regression (MVLR), Support Vector Regression (SVR) and Neural Network (NN)- (Numbers in Parenthesis are Percentage of Decrease in Errors in Comparison to the First Scenario (Just Have Electricity))

\begin{tabular}{l|l||l|l|l|l}
\hline Error & Methods & \multicolumn{1}{|c|}{ E } & \multicolumn{1}{|c|}{ E+W } & \multicolumn{1}{c}{ E+W+Tr.S } & E+W+Tr.N \\
\hline \hline & BSGP & 0.26152 & $0.239595(-9.15)$ & $0.239565(-9.17)$ & $0.22558(-15.93)$ \\
\cline { 2 - 6 } & ARIMAX & 0.32886 & $0.279485(-17.66)$ & $0.284505(-15.59)$ & $0.27786(-18.35)$ \\
\cline { 2 - 6 } SMAPE & MVLR & 0.399468 & $0.397163(-0.58)$ & $0.396761(-0.68)$ & $0.393566(-1.50)$ \\
\cline { 2 - 6 } & SVR & 0.418536 & $0.407971(-2.59)$ & $0.400147(-4.59)$ & $0.399571(-4.75)$ \\
\cline { 2 - 6 } & NN & 0.333198 & $0.332296(-0.27)$ & $0.328648(-1.38)$ & $0.323517(-2.99)$ \\
\hline \hline \multirow{5}{*}{ RMSE } & BSGP & 0.6133 & $0.5530(-9.83)$ & $0.5523(-9.94)$ & $0.5239(-14.57)$ \\
\cline { 2 - 6 } & ARIMAX & 0.6996 & $0.6066(-13.29)$ & $0.6164(-11.89)$ & $0.5787(-17.28)$ \\
\cline { 2 - 6 } & MVLR & 0.7759 & $0.7758(-0.02)$ & $0.7753(-0.08)$ & $0.7747(-0.16)$ \\
\cline { 2 - 6 } & SVR & 0.8220 & $0.8037(-2.23)$ & $0.7968(-3.07)$ & $0.7835(-4.68)$ \\
\cline { 2 - 6 } & NN & 0.6998 & $0.6898(-1.42)$ & $0.6735(-3.75)$ & $0.6686(-4.42)$ \\
\hline
\end{tabular}

Table 13. Error Table for Different Prediction Horizons for Electric Load Forecasting When All Input Variables Are Included (E+W+Tr.N)

\begin{tabular}{l||c|c|c|l||c|c|c}
\hline SMAPE & 1 day & 3 days & 7 days & \multicolumn{1}{|c|}{ RMSE } & 1 day & 3 days & 7 days \\
\hline \hline BSGP & 0.2256 & 0.2344 & 0.2458 & BSGP & 0.5239 & 0.5418 & 0.5588 \\
\hline ARIMAX & 0.2779 & 0.2845 & 0.3263 & ARIMAX & 0.5787 & 0.6218 & 0.6219 \\
\hline MVLR & 0.3936 & 0.3987 & 0.4636 & MVLR & 0.7747 & 0.7820 & 0.7823 \\
\hline SVR & 0.3996 & 0.3997 & 0.4579 & SVR & 0.7835 & 0.8031 & 0.8032 \\
\hline NN & 0.3235 & 0.3637 & 0.4229 & NN & 0.6686 & 0.7372 & 0.7757 \\
\hline
\end{tabular}

- By considering multi-source data fusion, in all the above methods, the error will be decreasing,

\section{CONCLUSION}

This article develops a traffic and load forecasting method by fusing the interdependencies among power consumption, traffic load, and weather information into a Bayesian spatiotemporal Gaussian Process framework. The proposed model identifies the most informative autocorrelation among the network and other covariates from different infrastructures, such as transportation and electricity networks, to capture the overall and local changes. The model is presented in a hierarchical structure and is inferred under the Bayesian framework. A real-life case study is conducted to demonstrate the efficacy of the proposed method using different scenarios based on the actual data from the city of Tallahassee, Florida. The results clearly showed that considering the interdependencies among power, transportation, and meteorological networks will help significantly improve traffic and electric load forecasting by $31 \%$ and $16 \%$ in the proposed method based on rMSEP and SMAPE criteria, respectively. Finally, our method is compared with some state-ofthe-art methods, i.e., ARIMAX, MVLR, SVR, and NN, and results demonstrated that the proposed method outperforms these methods. This study can be extended by incorporating other variables such as social and other city resources data such as water and gas. 


\section{ACKNOWLEDGMENTS}

The authors thank the City of Tallahassee and Michael Olsen and John Powell for their support and expertise. The contents of this article represent the authors' opinion and not the official view of the City of Tallahassee.

\section{REFERENCES}

Juan Aparicio, Justinian Rosca, Markus Mediger, Alexander Essl, Klaus Arzig, and Chris Develder. 2014. Exploiting road traffic data for very short term load forecasting in smart grids. In Proceedings of the 2014 IEEE PES Innovative Smart Grid Technologies Conference (ISGT'14) (Feb. 2014). DOI : https://doi.org/10.1109/ISGT.2014.6816498

Khandoker Shuvo Bakar. 2012. Bayesian Analysis of Daily Maximum Ozone Levels. Ph.D. Dissertation. University of Southampton.

Khandoker Shuvo Bakar and Sujit K. Sahu. 2015b. spTimer: Spatio-temporal bayesian modeling using R. F. Stat. Softw. 63, 15 (Feb. 2015), 1-32. http://www.jstatsoft.org/v63/i15/.

Khandoker Shuvo Bakar and Sujit K. Sahu. 2015a. spTimer: Spatio-temporal bayesian modeling using R. R package version 2.0-1. J. Stat. Softw. 63, 15 (Feb. 2015), 1-32. DOI : https://doi.org/10.18637/jss.v063.i15

U.S. Census Bureau. 2018. U.S. Census Bureau QuickFacts: Tallahassee city, Florida. Retrieved from https://www.census. gov/quickfacts/fact/table/tallahasseecityflorida/IPE120216.

Ervin Ceperic, Vladimir Ceperic, and Adrijan Baric. 2013. A strategy for short-term load forecasting by support vector regression machines. IEEE Trans. Power Syst. 28, 4 (Jul. 2013), 4356-4364. DOI: https://doi.org/10.1109/TPWRS.2013. 2269803

Mohamed Chaouch. 2014. Clustering-based improvement of nonparametric functional time series forecasting: Application to intra-day household-level load curves. IEEE Trans. Smart Grid 5, 1 (Sept. 2014), 411-419. DOI : https://doi.org/10.1109/ TSG.2013.2277171

Shin-Tzo Chen, David C. Yu, and A. R. Moghaddamjo. 1992. Weather sensitive short-term load forecasting using nonfully connected artificial neural network. IEEE Trans. Power Syst. 7, 3 (Aug. 1992), 1098-1105. DOI : https://doi.org/10.1109/59. 207323

T. W. S. Chow and C. T. Leung. 1996. Neural network based short-term load forecasting using weather compensation. IEEE Trans. Power Syst. 11, 4 (Nov. 1996), 1736-1742. DOI : https://doi.org/10.1109/59.544636

Mario Cools, Elke Moons, Lieve Creemers, and Geert Wets. 2010. Changes in travel behavior in response to weather conditions do type of weather and trip purpose matter? f. Transport. Res. Board 2157, 1 (Dec. 2010), 22-28. DOI: https:// doi.org/10.3141/2157-03

Jose Cordova, Lalitha Madhavi K. S., Ayberk Kocatepe, Yuxun Zhou, Eren E. Ozguven, and Reza Arghandeh. 2018. Combined electricity and traffic short-term load forecasting using bundled causality engine. (unpublished).

M. Davies. 1959. The relationship between weather and electricity demand. In Proceedings of the IEEE, Part C: Monographs (IEEE'59), Vol. 106. IET, 27-37. DOI: https://doi.org/10.1049/pi-c.1959.0007

Wen Deng, Hao Lei, and Xuesong Zhou. 2013. Traffic state estimaton and uncertainty quantification based on heterogeneous data sources: A three detector approach. Transport. Res. 57, 1 (Nov. 2013), 132-157. DOI : https://doi.org/10.1016/ j.trb.2013.08.015

Nour-Eddin El-Faouzi. 2010. Real-time Monitoring, Surveillance and Control of Road Networks Under Adverse Weather Conditions: Effects of Weather on Traffic and Pavement: State of the Art and Best Practices. INRETS.

Damien Fay and John V. Ringwood. 2010. On the influence of weather forecast errors in short-term load forecasting models. IEEE Trans. Power Syst. 25, 3 (Feb. 2010), 1751-1758. DOI : https://doi.org/10.1109/TPWRS.2009.2038704

Alan E. Gelfand. 2012. Hierarchical modeling for spatial data problems, spatial statistics. Spatial Stat. 1, 1 (Feb. 2012), 30-39. DOI : https://doi.org/10.1016/j.spasta.2012.02.005

Andrew Gelman, John B. Carlin, Hal S. Stern, David B. Dunson, Aki Vehtari, and Donald B. Rubin. 2004. Bayesian Data Analysis (3rd ed.). Chapman \& Hall/CRC.

Mahmoud Ghofrani, M. Hassanzadeh, Mehdi Etezadi-Amoli, and M. Sami Fadali. 2011. Smart meter based short-term load forecasting for residential customers. In Proceedings of the IEEE North American Power Symposium (NAPS'11), 99-118. DOI : https://doi.org/10.1109/NAPS.2011.6025124

Juan C. Herrera, Daniel B. Work, Ryan Herring, Xuegang Ban, Quinn Jacobson, and Alexandre M. Bayen. 2010. Evaluation of traffic data obtained via GPS-enabled mobile phones: The mobile century field experiment.Transport. Res. 18, 4 (Dec. 2010), 568-583. DOI : https://doi.org/10.1016/j.trc.2009.10.006

Aude Hofleitner, Ryan Herring, Pieter Abbeel, and Alexandre Bayen. 2012. Learning the dynamics of arterial traffic from probe data using a dynamic Bayesian network. IEEE Trans. Intell. Transport. Syst. 13, 4 (Dec. 2012), 1679-1693. DOI: https://doi.org/10.1109/TITS.2012.2200474 
Yu-Hsiang Hsiao. 2015. Household electricity demand forecast based on context information and user daily schedule analysis from meter data. IEEE Trans. Industr. Inf. 11, 1 (Oct. 2015), 33-43. DOI : https://doi.org/10.1109/TII.2014.2363584

Samuel Humeau, Tri Kurniawan Wijaya, Matteo Vasirani, and Karl Aberer. 2013. Electricity load forecasting for residential customers: Exploiting aggregation and correlation between households. Sustainable Internet and ICT for Sustainability (SustainIT). 1-6. DOI : https://doi.org/10.1109/SustainIT.2013.6685208

Jarod C. Kelly, Tulga Ersal, Chiao-Ting Li, Brandon M. Marshall, Soumya Kundu, Gregory A. Keoleian, Huei Peng, Ian A. Hiskens, and Jeffrey L. Stein. 2015. Sustainability, resiliency, and grid stability of the coupled electricity and transportation infrastructures: Case for an integrated analysis. f. Infrastruct. Syst. 21, 4 (Feb. 2015), 04015001.

Weicong Kong, Zhao Yang Dong, David J. Hill, Fengji Luo, and Yan Xu. 2017. Short-term residential load forecasting based on resident behaviour learning. IEEE Trans. Power Syst. 33, 1 (Mar. 2017), 1087-1088. DOI : https://doi.org/10.1109/ TPWRS.2017.2688178

Lalitha Madhavi K. S., Mostafa Gilanifar, Yuxun Zhou, Eren E. Ozguven, and Reza Arghandeh. 2018. Multivariate deep causal network for time series forecasting in independent networks. In Proceedings of the 57th IEEE Conference on Decision and Control.

Sharad Kumar, Shashank Mishra, and Shashank Gupta. 2016. Short term load forecasting using ANN and multiple linear regression. In Proceedings of the Second International Conference on Computational Intelligence and Communication Technology (CICT'16). DOI : https://doi.org/10.1109/CICT.2016.44

K. S. Lalitha Madhavi, Jose Cordova, Mehmet Baran Ulak, Michael Ohlsen, Eren E. Ozguven, Reza Arghandeh, and Ayberk Kocatepe. 2017. Advanced electricity load forecasting combining electricity and transportation network. In Proceedings of the IEEE Conference of the 2017 North American Power Symposium (NAPS'17). DOI : https://doi.org/10.1109/NAPS.2017. 8107312

K. S. Lalitha Madhavi, Mostafa Gilanifar, Yuxun Zhou, Eren E. Ozguven, and Reza Arghandeh. 2019. Causal Markov Elman network for load forecasting in multi network systems. IEEE Trans. Industr. Electr. 66, 2 (Feb. 2019), 1434-1442. DOI : https://doi.org/10.1109/TIE.2018.2851977

Benjamin Marti. 2006. Integrated Analysis of Energy and Transportation Systems. Master's thesis. Swiss Federal Institute of Technology (ETH) Zurich, Zurich.

Bertil Matern. 1986. Spatial Variation (2nd ed.). Springer-Verlag, New York.

Alfredo Nantes, Dong Ngoduy, Ashish Bhaskar, Marc Miska, and Edward Chung. 2016. Real-time traffic state estimation in urban corridors from heterogeneous data. Transport. Res. 66, 1 (May 2016), 99-118. DOI : https://doi.org/10.1016/j.trc. 2015.07.005

Hung Nguyen and Christian K. Hansen. 2017. Short-term electricity load forecasting with time series analysis. In Proceedings of the 2017 IEEE International Conference onPrognostics and Health Management (ICPHM'17). DOI: https://doi.org/ 10.1109/ICPHM.2017.7998331

Julien Ostermann and Falko Koetter. 2016. Energy-management-as-a-service: Mobility aware energy management for a shared electric vehicle fleet. In Proceedings of the 2016 th International Conference on Smart Cities and Green ICT Systems (SMARTGREENS'16).

Davide Pinzan, Ayberk Kocatepe, Mostafa Gilanifar, Mehmet B. Ulak, Eren E. Ozguven, and Reza Arghandeh. 2018. Datadriven and hurricane-focused metrics for combined transportation and power networks resilience. In Proceedings of the 2018 Transportation Research Board Annual Meeting (TRB'18).

Christian Rudloff, Maximilian Leodolter, Dietmar Bauer, Roland Auer, Werner Brög, and Knud Kehnscherper. 2015. Influence of weather on transport demand: A case study from the Vienna region. F. Transport. Res. Board 24821, 1 (Jan. 2015), 110-116. DOI : https://doi.org/10.3141/2482-14

Slobodan Ruzic, Aca Vuckovic, and Nikola Nikolic. 2003. Weather sensitive method for short term load forecasting in electric power utility of serbia. IEEE Trans. Power Sys. 18, 4 (Nov. 2003), 1581-1586. DOI : https://doi.org/10.1109/TPWRS 2003.811172

Rijurekha Sen, Andrew Cross, Aditya Vashistha, Venkata N. Padmanabhan, Edward Cutrell, and William Thies. 2013. Accurate speed and density measurement for road traffic in India. In Proceedings of the 3rd ACM Symposium on Computing for Development (ACM DEV'13). ACM, 1-10. DOI : https://doi.org/10.1145/2442882.2442901

James W. Taylor and Roberto Buizza. 2002. Neural network load forecasting with weather ensemble predictions. IEEE Trans. Power Syst. 17, 3 (Nov. 2002), 626-632. DOI : https://doi.org/10.1109/TPWRS.2002.800906

WeatherSTEM. 2018. WeatherSTEM.Leon County. Retrieved from https://leon.weatherstem.com.

Young-Min Wi, Sung-Kwan Joo, and Kyung-Bin Song. 2012. Holiday load forecasting using fuzzy polynomial regression with weather feature selection and adjustment. IEEE Trans. Power Syst. 27, 2 (Dec. 2012), 596-603. DOI : https://doi.org/ 10.1109/TPWRS.2011.2174659

Daniel B. Work, Sebastien Balndin, Olli-Pekka Tossavainen, Benedetto Piccoli, and Alexandre M. Bayen. 2010. A traffic model for velocity data assimilation. Appl. Math. Res. Express (Apr. 2010), 1-35. DOI : https://doi.org/10.1093/amrx/ abq002. 
Tao Xing, Xuesong Zhou, and Jeffrey Taylor. 2013. Designing heterogeneous sensor networks for estimating and predicting path travel time dynamics: An information-theoretic modeling approach. Transport. Res. 57, 1 (Nov. 2013), 66-90. DOI : https://doi.org/10.1016/j.trb.2013.09.007

Desheng Zhang, Juanjuan Zhao, Fan Zhang, Tian He, Haengju Lee, and Sang H. Son. 2017. Heterogeneous model integration for multi-source urban infrastructure data. ACM Trans. Cyber-Phys. Syst. 1, 1 (Feb. 2017). DOI : https://doi.org/10.1145/ 2967503

Yu Zheng, Licia Capra, Ouri Wolfson, and Hai Yang. 2014. Urban computing: Concepts, methodologies, and applications. ACM Trans. Intell. Syst. Technol. 5, 3 (Sept. 2014). DOI : https://doi.org/10.1145/2629592

Received December 2017; revised October 2018; accepted November 2018 